\title{
Wireless Device Location Sensing In a Museum Project
}

Tanvir Anwar

Sydney, Australia

Email: tanvir.anwar.australia@gmail.com

\section{Abstract}

Indoor navigation can be implemented with Bluetooth technology as sensors. We use RTT (Round Trip Time) as an attribute towards the navigation of an object. Designing the scheme for indoor networks with the introduction of pre-fixed co-ordinates has been used as a database. This paper presents a new algorithm that incorporate real life signal strength measurement from access point and peers to estimate position and distance using Bluetooth sensor network. The main feature of this paper is to see how RTT behaves with distance and then what approach we must take to make RTT more robust. We conducted several experiments to validate our proposed algorithm and study RTT behavior in real life application.

Keywords: Round Trip Time (RTT), Receive Signal Strength Indicator (RSSI), Bluetooth tags, Sensor

\section{INTRODUCTION}

Location awareness is becoming the most important issue in many cases, such as when an urgent accident needs the nearest doctor in the hospital, or in the university the staff members want to check available members for a quick meeting, or even to track an object. These are some of the important questions which certainly need fast, reliable answers by keeping the privacy as a priority. For various reasons localization is rapidly growing and context awareness consists of six questions involving what, who, where, when, why and how? Location awareness mainly tries to answer two of them: "where" and "who". Location sensing can be divided into indoor and outdoor awareness. Indoor systems mainly contain a set of antennas with wireless tags. The antennas communicate wirelessly with tags, which are

\author{
Dr. Priyadarsi Nanda \\ School of Computing and Communications \\ Faculty of Engineering and IT \\ University of Technology, Sydney, Australia \\ Email: priyadarsi.nanda@uts.edu.au
}

attached to humans, or to desired position of the objects. Mobile handsets are also often personal in nature, so the statistics related to measurements reflect behavioral patterns of the user or object. We demonstrate how prototypical patterns of behavior may be found in data produced by Bluetooth scanning. It appears that cyclical nature of the patterns reflects well various daily routines of test objects. The scanning results are then sent via GPRS/AP to a backend server for further processing for a fixed duration of time. In this project we monitor various data received to ensure that all palmtops and Bluetooth dongles attached to every item of the museum are operating properly. The data set consists of phone ID, timestamp, and MAC address. Some of detected MAC address corresponds to known Iocations (location of every items of museum). Location evaluation is performed by sensing Bluetooth signal strength with a reference model based approach. We discuss problems which arise when the Bluetooth Received Signal Strength Indicator (RSSI) is used as a measurement of signal strength and propose a novel access point technique based on such data. This access point allows the reading of a wider range of signal strengths using RSSI [1] [2].

This paper is organized into seven sections. Section I gives the introduction. Section II presents an overview of the architecture while section III identifies parameters making RTT as a strong candidate for improvement on localization technique. Section IV and V presents various aspects of Bluetooth protocol and how the database can be accessed. Our proposed algorithm and results of the experiments are given in Section VI. We conclude our work in Section VII. 


\section{SOFTWARE ARCHITECTURE AND IMPLEMENTATION}

Our architecture is inspired by the location stack and reduce the interaction between separate layers.Figure-1 below illustrates the architecture.

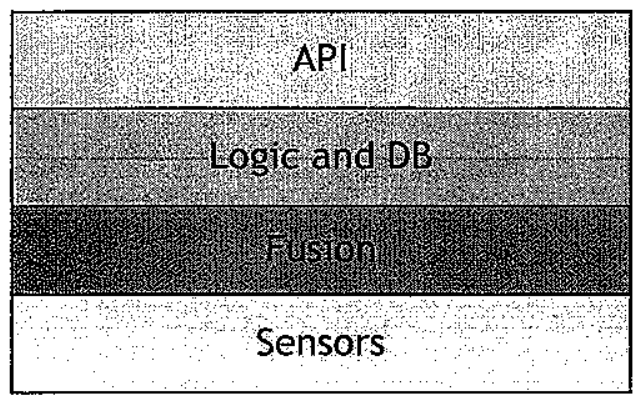

\section{Figure1: Schematic diagram of the layered Architecture}

\section{A. Sensor layer}

This layer is divided into two modules: sensor driver and sensor data abstractor. The former provides low-level software that gathers raw sensor data from the corresponding sensor hardware. The native sensor data is sent to a sensor abstractor, which runs in location server process. These abstractors optionally have access to a database to query the physical or semantic location of a Tag reader or a Bluetooth host [6].

\section{B. Fusion layer}

The fusion layer takes data from all sensors in the uniform format used by the sensor layer and calculates a single resulting position. This layer can implement algorithms based on individual requirements. Algorithms with very high precision and high requirements in computing power are available, as well as simpler and faster algorithms, which can handle a much higher number of simultaneous tracked clients.

\section{Logic and database layer}

The logic and database layer maintains databases for all tracked objects and their environment. It can store the actual semantic or geometric position and the history of locations (if desired) of the tracked objects. Contextual information like names, room numbers and other properties are also located at this layer. Digital representations of the environment are stored as a hierarchal zone model, whereas the logic layer can look up a geometric or semantic position in the zone database to get additional symbolic information like the room or area in which the position is located [6].

\section{API layer}

The API layer describes the interface of our service used, to obtain position and contextual information as well as basic relationships like containment, and proximity, which we implemented as common and expandable set of features. The API is used both for client/server communication and for communication between the servers [6].

\section{ROBUSTNESS OF RTT}

We used RTT, the time a packet takes to travel to the receiver and then to come back to the transmitter. Three main components of RTT consists of: processing time, flight time and serialization delay. In Bluetooth devices we have several layers. In order to make Bluetooth working as a good navigator the link manager protocol, L2CAP layer and BNEP are being used.

The link manager protocol that is stored in the firmware of the Bluetooth holds protocols of inquiry, inquiry scan paging and few other necessary uni-directional protocols. They are needed to discover the device present in the range of Bluetooth and then create the link among the Bluetooth enabled devices. According to the standard, one of the available Bluetooth enabled devices, has to agree to be a master of the piconet (one piconet can have maximum of one master and seven slaves). Once the link is established, the immediate higher layer L2CAP protocols are implemented. This layer does the function of data management. The size of packet, the sequence of transmission, encapsulation of higher layers, are taken care of in this L2CAP section. The size of the packet, serialization delay and the speed of the packet transmission are closely related to the media of packet transmission.

Though, with the available mathematical and logical knowledge, we can draw conclusions of the performance of the above parameters, yet the theoretical facts can be established throught experimental observations [3] [4]. The readings of RTT versus distance inside and outside the 196 
help us analyze the nature of transmission for the packets of fixed size.

What is prominent in the readings is the absence of RTT values for some distances. As mentioned before, RTT is nothing but, the time taken for packets of a fixed size, to travel from the source to the destination and back to the source. The absence of RTT, in the readings does not establish this fact, because packets are lost. The inconsistency of RTT with distance is also conspicuous from the graph. To increase the robustness of the data (RTT), we only consider the cluster of readings very close to each other and get rid of the abnormal readings with high variance, from the cluster of ten sampled readings for each distance. The reading which has a high variance, and is very far from the cluster will have bad effects on the rest of the readings of the specified cluster.

Like GSM system for mobile, interior Bluetooth location sensing system network also consists of many small cells. We make each cell as one piconet, having one master/Gateway to the rest of the network (in our case, Bluetooth enabled network access point) at the centre of each cell and many other slaves (any other mobile Bluetooth enabled devices).

\section{DATABASE IN BLUETOOTH}

Unlike the home agent in mobile networks, the home agent in Bluetooth networks mostly involves handling of the database and the handover process. To present the information

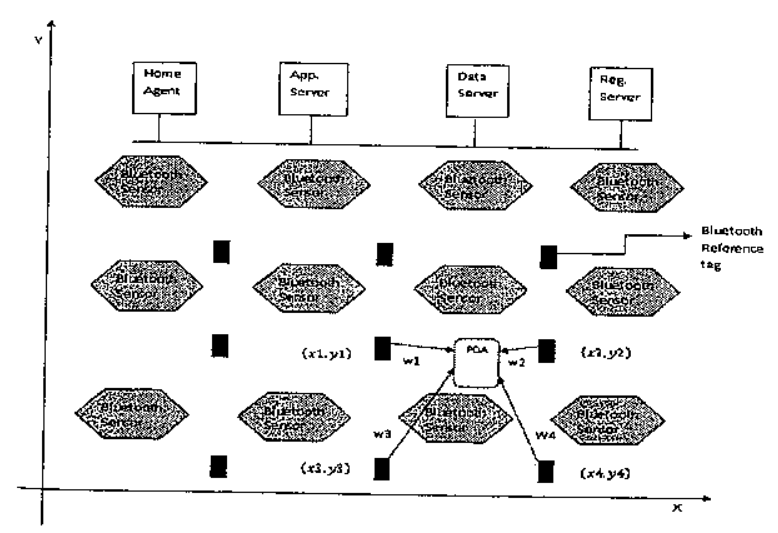

Figure 2: The scheme with tags and sensors (in our case, the information of different objects in the museum) in the best possible way, the management of the database within the system is taken care of very delicately. To build our database we need to start with an experiment with predefined co-ordinates for some Bluetooth enabled devices considered as reference tags constantly emitting packets to travel to the nearest access point (in our case, sensors). The sensors are also located at a fixed co-ordinate. Though these packets emitted from one such tag are received by many sensors, yet we will be able to come up with a limited number of neighboring sensors by setting a common threshold E value( see the Euclidian distance formula ) for every bluetooth enabled tag. The scheme is presented in Fig 2.

We created a sample database called "Museum Database" with attributes describing: object number, corresponding co-ordinate of the object and related information of the object. RTT (Round Trip Time) of each tag is sensed by four nearest neighbors (combination of four neighboring sensors each with a RTT value above a certain high threshold corresponds to one reference tag). The correlation between the mobile Bluetooth enabled device and the reference tag is calculated using the Euclidian distance formula [3].

$$
E_{i}=\sqrt{\sum_{k=1}^{n}\left(r_{i, k}-u_{k}\right)^{2}}
$$

The least value of $E$ allows us to find the closest reference tag to the mobile Bluetooth device. The location of the reference tag is the location of the object in the museum. In the formula $r$ and $\mathrm{u}$ are the RTT (Round Trip Time) values of reference tag and the mobile Bluetooth enabled device respectively. Every sensor in the network senses these RTT values of the reference tags [3]. Ethernet header is replaced with a BNEP header at the interface.

\section{BLUETOOTH LAYER PROTOCOLS}

As shown in Figure-2, two different kinds of communication are established involving the device. The first one is wireless (between two Bluetooth enabled devices) and the second one is wired. In terms of protocol layer, in wireless 
communication it is the BNEP header which holds the Ethernet payload, whereas in wired communication it is the Ethernet header that holds the Ethernet payload. So whenever we have packets transferred between two Bluetooth enabled devices it is the BNEP header that holds the payload, otherwise it is Ethernet header for conventional communication. We present the network infrastructure implemented in the lab with the following example. A wired networked device (Device $C$ ) communicates with a mobile Bluetooth enabled device(Device $A$ ) via a Bluetooth enabled NAP (Device B), Device B will act as a NAP (Network Access Point) and remain as a piconet master for the Bluetooth network, which is connected via a wired network to Device C.

For every network communication irrespective of the wire, before a packet can be transferred between two devices, a bridge is made and the Bluetooth packets are divided into several smaller baseband packets before they can be transmitted. Such task is performed by the L2CAP protocol layer. L2CAP provides connection oriented or connectionless data channels. The physical link is the link that is established between the master and slave units. There are two different kinds of link defined in the Bluetooth specifications:

- Synchronous Connection-Oriented link, or SCO -link

- Asynchronous Connection-Less link, or ACL-link

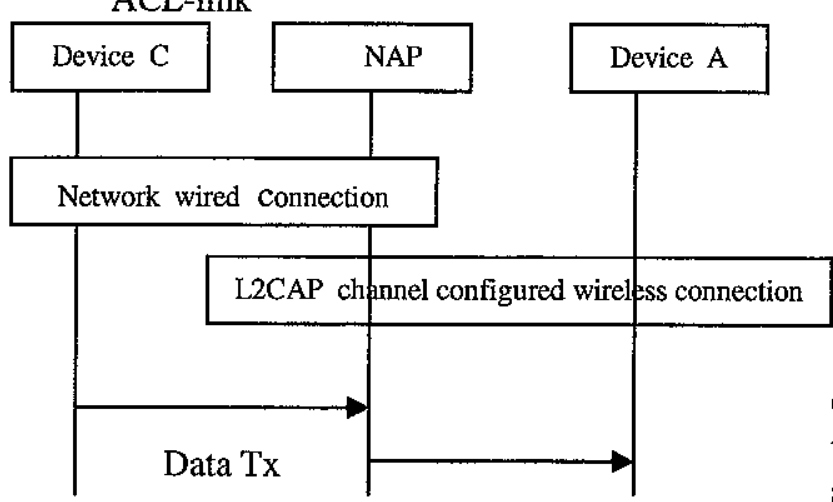

Data Tx

Figure 3: Wireless Device Protocol stack (Network Access Point)

The functional requirements for Bluetooth Network Encapsulation protocol (BNEP) include the support of Personal Area Networking (PAN) profile.

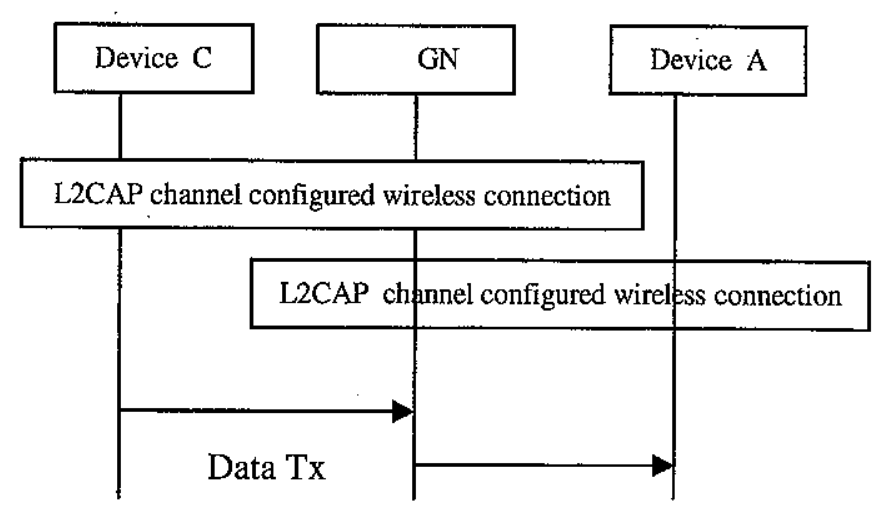

Data Tx

\section{Figure 4: Wireless Device Protocol stack (Gateway Network)}

There are three kinds of PAN profile and they entirely depend upon the topology in which the personal area network is setup. Each of these three profiles has different roles. A simple mobile Bluetooth enabled device in a piconet that avail the network wirelessly through either a Bluetooth Network Access Point connected to internet or a central home agent or through a Group Ad-Hoc Networking Controller called PANU. So PANU is a client. GN and NAP are service providers. But there is a basic difference between these two. NAP is connected to the larger network infrastructure with wire whereas, GN is like any other mobile Bluetooth enabled device acting as a packet forwarding node that can serve up to seven slaves[5][7][8].

\section{BLUETOOTH NAVIGATOR}

\section{ALGORITHM AND RESULTS}

Collecting all the RTT values of one moving unknown tag sensed by all the neighboring sensors to a server called main server which holds the program to manipulate this database of RTT values with the already existing database of RTT values of all the reference tags. These RTT values will be stored into a file where my program will fetch the value from when necessary. There is a file containing the coordinate values and their corresponding reference tag RTT values. After this we calculate all the E 
values implementing the equation mentioned above between one unknown tag against every other reference tag. Then we set a threshold value $\mathrm{k}(\mathrm{k}$ is a very small number and is small enough to satisfy our criteria.). Any $\mathrm{E}$ value below $\mathrm{k}$ is to be taken into account and others are to be discarded. Now we can fetch the coordinate of the reference tags associated with those $E$ values that are below a certain threshold value $\mathrm{k}$.

We calculate the weights associated only with those reference tags whose $\mathrm{E}$ values are below the threshold value $\mathrm{k}$. Finally we calculate the co-ordinate of the moving palm top device. Unknown tag using the co-ordinate geometry formula is presented below [3].

$$
\begin{aligned}
x_{\text {unknown }} & =\frac{1}{2}\left(x_{\text {nearest } 1}+x_{\text {nearest } 2}\right) \\
y_{\text {unknown }} & =\frac{1}{2}\left(y_{\text {nearest } 1}+y_{\text {nearest } 2}\right) \\
(x, y) & =\frac{1}{k} \sum_{i=1}^{k}\left(x_{r i}, y_{r i}\right) \\
(x, y) & =\sum_{i=1}^{k} w_{i}\left(x_{r i}, y_{r i}\right)
\end{aligned}
$$

$\mathrm{W}_{\mathrm{i}}$ is the function of the $\mathrm{E}$ of all $\mathrm{K}$ nearest neighbors. Our approach of the weight depends on the $\mathrm{E}$ as:

$$
w_{j}=\frac{\frac{1}{E_{i}}}{\sum_{i=1}^{k} \frac{1}{E_{i}}}
$$

In this approach, the reference tag with the smallest $E$ value has the largest weight.

\begin{tabular}{|c|c|c|c|}
\hline Dist(m) & year & Time(ms) & $\mathrm{Avg}(m)$ \\
\hline \multirow{3}{*}{1} & 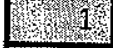 & 3908 & \\
\hline & 2 & 4770 & \\
\hline & ry & 5553 & \\
\hline \multirow{3}{*}{2} & reve & 2770 & \\
\hline & 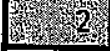 & 3481 & \\
\hline & (3) & 4124 & \\
\hline \multirow{3}{*}{3} & (5) & 1967 & \\
\hline & (2) & 2997 & \\
\hline & & 3196 & \\
\hline \multirow{3}{*}{4} & 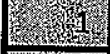 & 2024 & \\
\hline & 的 & 3151 & \\
\hline & (3) & 3337 & \\
\hline \multirow{3}{*}{5} & $y$ & 3025 & \\
\hline & 2 & 4153 & \\
\hline & W & 4786 & \\
\hline \multirow{3}{*}{6} & 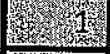 & 9120 & \\
\hline & 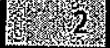 & 10834 & \\
\hline & 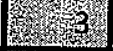 & 13152 & \\
\hline \multirow{3}{*}{7} & $y_{4}$ & 2906 & \\
\hline & 2. & 3716 & \\
\hline & - & 4273 & \\
\hline \multirow{3}{*}{8} & rescis & 4161 & \\
\hline & $\begin{aligned} 4 \\
3\end{aligned}$ & 4998 & \\
\hline & 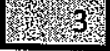 & 6667 & \\
\hline
\end{tabular}

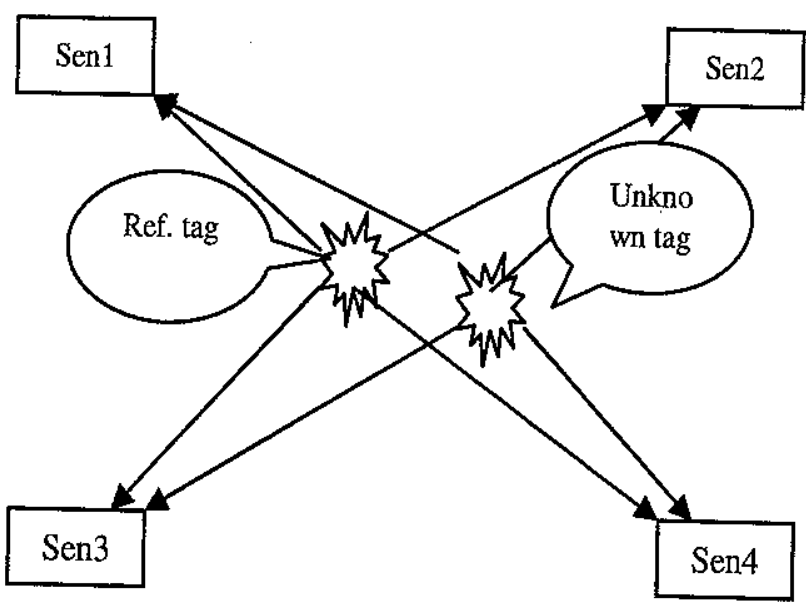

Figure 5: Algorithm implemented to locate unknown tag

Experimental results for inside lab: 


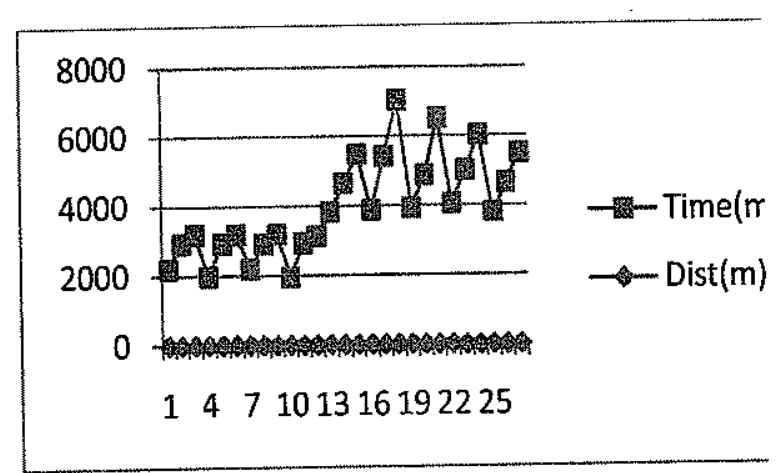

Figure 6: RTT vs Distance (inside lab)

Experimental results outside Lab:

\begin{tabular}{|c|c|c|c|}
\hline $\operatorname{Dist}(m)$ & $\mathrm{SSeq}_{\mathrm{S}}$ & Time(ms) & $\mathrm{Avg}\left(\mathrm{ns}_{\mathrm{N}}\right.$ \\
\hline \multirow{3}{*}{1} & & 2204 & \\
\hline & & 2940 & \\
\hline & & 3188 & \\
\hline \multirow{3}{*}{2} & & 1985 & \\
\hline & & 2931 & \\
\hline & & 3190 & \\
\hline \multirow{3}{*}{3} & & 2192 & \\
\hline & & 2923 & \\
\hline & & 3197 & \\
\hline \multirow{3}{*}{4} & & 1956 & \\
\hline & & 2942 & \\
\hline & & 3140 & \\
\hline \multirow{3}{*}{4.5} & & 3824 & \\
\hline & & 4651 & \\
\hline & & 5487 & \\
\hline \multirow{3}{*}{6} & & 3879 & \\
\hline & & 5429 & \\
\hline & & 7039 & \\
\hline \multirow{3}{*}{6.5} & & 3924 & \\
\hline & & 4879 & \\
\hline & & 6516 & \\
\hline \multirow{3}{*}{7} & & 4043 & \\
\hline & & 5015 & \\
\hline & 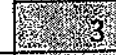 & 6028 & \\
\hline \multirow{3}{*}{7.5} & & 3805 & \\
\hline & 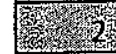 & 4655 & \\
\hline & 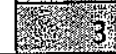 & 5497 & \\
\hline
\end{tabular}

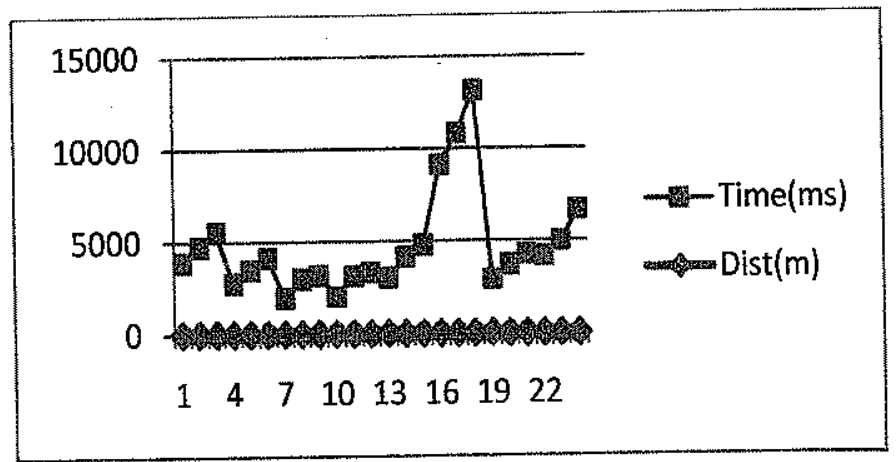

Figure 7: RTT vs Distance (outside lab)

\section{CONCLUSION:}

The experimental results suggest it is possible to use RTT based measurements for location based services using Bluetooth. Though our result is not linear, yet such variation with distance can be of great use. More work can be done on data link layer to acknowledge the packet received or make the payload shorter to make RTT even more robust in location based navigation system.

\section{ACKNOWLEDGMENT}

I acknowledge the effort from Mr,Terry, Dr, Priyadarsi, Zhiyuan Tan with their thoughts, ideas and also with the experiment.

\section{REFERENCES}

[1] Rathi S, Bluetooth Protocol Architecture, Dedicated Systems Magazine, Microwave System Corporation, Q4.28-33, 2000

[2] Capone A, Geria M, Kapoor R, Efficient Polling. Schemes for Bluetooth Picocells, IEEE International Conference on Communications (ICC), 2001, Vol. 7, page: 1990 - 1994

[3] Patil A.P, Performance of Bluetooth Technology and their Applications to location sensing, Master's thesis,MSU, 2002

[4] Song O, Dong-Young K, Chong-Ho C, HyunSang J, Fast Handover for Bluetooth Network Access Systems, IEEE International conference on communications, Alaska, USA,2003

[5] Bluetooth Personal Area Networking Profile, February 14, 2003

[6] Roder P, Hoffmann M, Ritscher M, A distributed framework for location sensing systems, Institute Secure Tele cooperation, Darmstadt, Germany 2003

[7] Bluetooth Network Encapsulation Protocol (BNEP) Specification, Bluetooth SIG, Inc., 2001

[8] Bluetooth Protocol stacks Overview, Palo Wireless, Bluetooth SIG Inc, Tutorial 2001 
Subject: NTC 2010: Acceptance of Full Paper

From: "Jackie Chan on behalf of Dr. Tony Luo" <jackie@networkcomm.org>

Date: Mon; 11 Oct 2010 18:39:05 +0800

To: tanvir.anwar.australia@gmail.com, Priyadarsi.Nanda@uts.edu.au

\section{Annual International Conference on Network Technologies \& Communications NTC 2010 \\ 29 - 30 November 2010, Phuket Beach Resort, Thailand www.networkcomm.org}

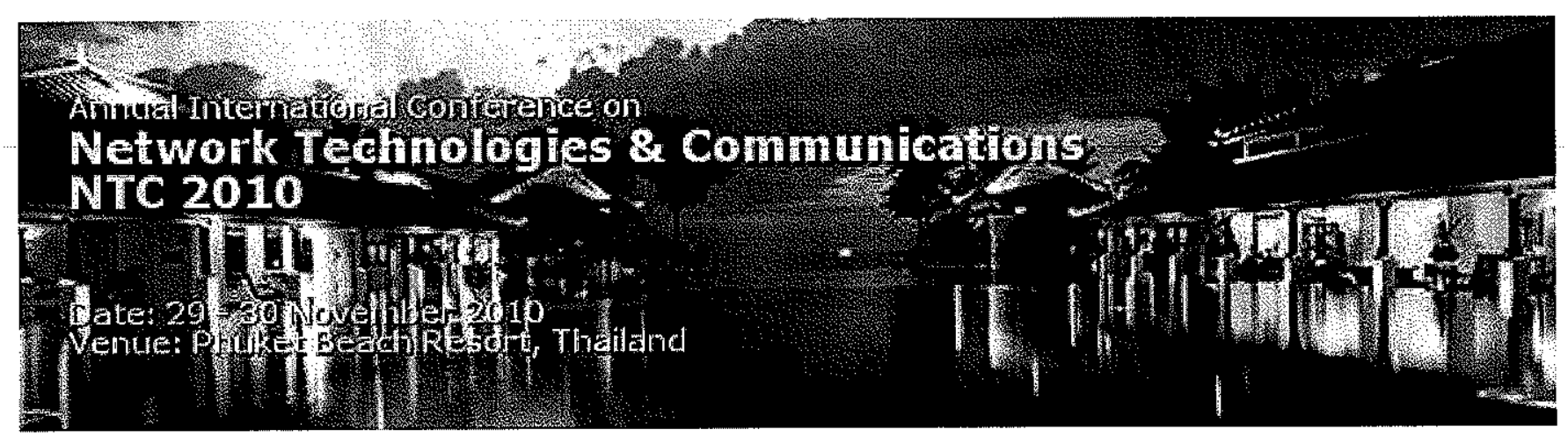

Paper Code: 35

Paper Title: Wireless Device Location Sensing in a Museum Project

Dear Author(s),

We are pleased to inform you that your paper as referenced above has been accepted for presentation at NTC 2010 and for publication in the conference proceedings. Congratulations!

In order for your paper to be published, you are required to complete the registration where the instructions are available at conference website (visit http://www.networkcomm.org/ and click on "Registration" on the left panel). Kindly note that the Early-bird Registration Deadline is November 1, 2010.

In addition, we would like to share with you some highlights of the conference:

- The Conference Proceedings will be indexed by / included in CiteSeerX SCIrus, EBSCO, getCITEDand Google Scholar. In addition, they will be submitted to IEEE xplore, ACM Digital Library El Compendex, and ISTP for indexing/inclusion subject to acceptance / approval.

- Selected papers will be published in GSTF International Journal on Computing (JoC) and GSTF associated journals subject to extension/acceptance.

- Best Paper Awards and Best Student Paper Awards will be conferred at the conference (in order to qualify for the award, the paper must be presented at the conference).

Keynote Address:

Knowledge-based Support throughout the Product Life Cycle

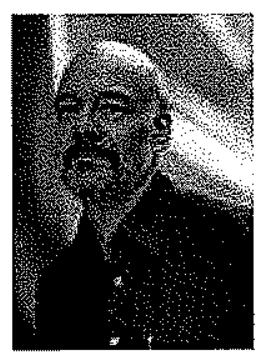

\section{Prof. Peter Struss}

Head, MQM research group

(Model-Based Systems \& Qualitative Reasoning)

Technical University of Munich, Germany.

[ Brief Profile]

The conference will be held at Phuket Beach Resort. You may want to take a look at the official website of the Phuket Tourism Board.

If you need any assistance pertaining to Visa application and/or hotel accommodation, etc., please do not hesitate to contact us at: 
NTC Conference Secretariat:

Email: info@networkcomm.org

Tel: +65 63270166

Fax: +65 63270162

Warm Regards,

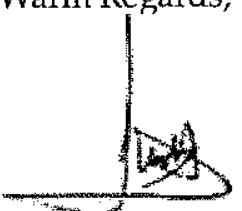

Dr. Tony Luo, Organizing Co-Chair, Email: tony@globalstf.org

International Conference on Network Technologies \& Communications (NTC) www.networkcomm.org

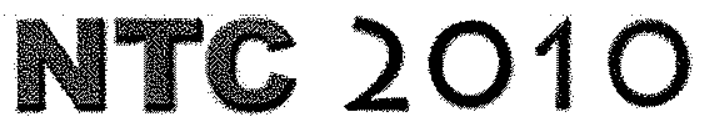

Network Technologies \& Communications 
Subject: RE: NTC 2010: Acceptance of Full Paper

From: "NTC Conference Secretariat" <jackie@networkcomm.org>

Date: Mon, 8 Nov 2010 15:55:22 +0800

To: "'Priyadarsi Nanda'" <pnanda@it.uts.edu.au>

Dear Priyadarsi,

Apologies for the delayed response.

Please find attached the review comments provided by the TPC.

As such, we would require that the necessary changes are incorporated and send us your revised paper as per the recommendations of the reviewer in camera-ready format no later than 15 November 2010.

Our editorial part will also provide assistance, if needed with regards to grammar only.

Should you require further assistance, please do not hesitate to contact me.

Best Regards,

Jackie Chan (MS.)

NTC Conference Secretariat

wWw. networkcomm. org

-----Original Message------

From: Priyadarsi Nanda [mailto:pnandalit.uts.edu.au]

Sent: Tuesday, October 19, 2010 8:16 AM

To: Jackie Chan on behalf of Dr. Tony Luo

Cc: tanvir.anwar.australialgmail.com; Priyadarsi.Nanda@uts.edu.au

Subject: Re: NTC 2010: Acceptance of Full Paper

Dear Jackie,

I am still waiting for your reply. The reviewer's comments on our paper

and camera ready copy instructions are very important for us to register

for the conference. Can you please send them to us ASAP.

looking forward to your reply.

Regards

Priyadarsi

On 13/10/2010 2:20 PM, Priyadarsi Nanda wrote:

Thanks for accepting our paper. Can you send us the reviewer's comments and also information on how to prepare the camera ready copy for the

conference. Also is it mandatory to present the paper in the conference by one of the author?

Looking forward to hear from you.

Regards

Priyadarsi

On 11/10/2010 9:39 PM, Jackie Chan on behalf of Dr. Tony Luo wrote:

*Annual International Conference on Network Technologies \&

Communications*

*NTC 2010*

*29** - 30 November 2010, Phuket Beach Resort, Thailand*

www. networkcomm.org <http://www. networkcom.org >

ntc ban. JPG

Paper Code: 35

Paper Title: Wireless Device Location Sensing In a Museum Project

Dear Author(s), 
We are pleased to inform you that your paper as referenced above has been accepted for presentation at $\mathrm{NTC} 2010$ and for publication in the conference proceedings. Congratulations!

In order for your paper to be published, you are required to complete the registration where the instructions are available at conference website (visit http://Www.networkcomm.org/ and click on "Registration" on the left panel). Kindly note that the Early-bird Registration Deadline is November 1, 2010.

In addition, we would like to share with you some highlights of the conference:

* The Conference Proceedings will be indexed by / included in

*CiteSeerX* <http://citeseerx.ist.psu.edu/ $\geq$, *SCIrus*

<http://www.scirus.edu/ $r^{*} * \star E B S C O * \leq h t t p: / / w w w . ~ e b s c o h o s t . c o m />$,

*getCITED* <http://WWW.getcited.org/>and *Google Scholar*

<http://scholar.google.com.sg/>. In addition, they will be

submitted to *IEEE xplore $\langle$ http://ieeexplore.ieee.org/>,****ACM

Digital Library <http://portal.acm.org/dl.cfm>*, *EI Compendex

<http://www.ei.org/>*, and *ISTP

<http://www.dimdi.de/static/en/db/dbinfo/ii78.htm>* for

indexing/inclusion subject to acceptance / approval.

* Selected papers will be published in GSTF International Journal on

Computing (JoC) 〈http://www.globalstf.org/journal-computing.php>

and GSTF associated journals subject to extension/acceptance.

* *Best Paper Awards* and *Best Student Paper Awards* will be

conferred at the conference (in order to qualify for the award,

the paper must be presented at the conference).

- Keynote Address: *

*Knowledge-based Support throughout the Product Life Cycle** *

http://itsecurityconf.org/images/photo prof struss.jpg

\section{*Prof. * *Peter Struss*}

Head, MQM research group

(Model-Based Systems \& Qualitative Reasoning)

Technical University of Munich, Germany.

[ Brief Profile <http://itsecurityconf.org/biol.html> ]

- The conference will be held at *Phuket Beach Resort*. You may want to take a look at the official website of the Phuket Tourism Board

<http://www. yoursingapore.com/content/traveller/en/experience.html>.

If you need any assistance pertaining to Visa application and/or hotel accommodation, etc., please do not hesitate to contact us at:

NTC Conference Secretariat:

Email: infodnetworkcomm.org <mailto:info@networkcomm.org>

Tel: +6563270166

Fax: +65 63270162

Warm Regards,

tonysignature.JPG

Dr. Tony Luo, Organizing Co-Chair, Email: tonyeglobalstf.org

<mailto: tony@globalstf.org >

*International Conference on $* *$ Network Technologies \& Communications $(\mathrm{NTC}) * *$

*WWw. networkcomm.org shttp: //Www. networkcomm.org $>$

ntc logo.png 
$-$

Dr.Priyadarsi Nanda, PhD (Computing Science)

Senior Lecturer

Program Leader, Bachelor of Information Technology (BIT)

School of Computing and Communications

Faculty of Engineering and IT

University of Technology, Sydney, Australia

PO BOX: 123, Broadway, NSW-2007

Office: CB10.04.432

Tele: +61-2-95141853

Fax: $+61-2-95141807$

URL: wWw-staff.it.uts.edu,au/ pnanda/

CRICOS provider code: 00099F

\begin{tabular}{|l|l|l|}
\hline NTC Paper ID 35.docx & \begin{tabular}{l} 
Content-Type: $\begin{array}{c}\text { application/vnd.openxmlformats- } \\
\text { officedocument.wordprocessingml.document } \\
\text { Content-Encoding: base64 }\end{array}$ \\
\hline
\end{tabular} \\
\hline
\end{tabular}




\section{Paper Title: Wireless Device Location Sensing In Mesuem Project}

This paper integrate the object oriented metrics used to determine the testability of the software into a single overall value called the Precise Testability Index used to find out the testability efforts for the software project.

Fit to be a graduate project, no research contribution. Some referenced material directly lifted from the papers: e.g. Unknown tag using the co-ordinate geometry formula that is mentioned at the end part of this chapter [7]. ) Why chapter is mentioned???

Some set of experiments and readings.

No Experimentation Details given.

No Research Novelty.

Many references are not in complete format, some are missing publisher, some date and venue

Areas of improvement:

Research Inclination is required.

Proper Experiment platform and framework should be discussed.

Lot of sentences need to be revised

Paper needs to be improved. 


\section{Paper Title: Wireless Device Location Sensing In Mesuem Project}

This paper integrate the object oriented metrics used to determine the testability of the software into a single overall value called the Precise Testability Index used to find out the testability efforts for the software project.

Fit to be a graduate project, no research contribution. Some referenced material directly lifted from the papers: e.g. Unknown tag using the co-ordinate geometry formula that is mentioned at the end part of this chapter [7].) Why chapter is mentioned???

Some set of experiments and readings.

No Experimentation Details given.

No Research Novelty.

Many references are not in complete format, some are missing publisher, some date and venue

Areas of improvement:

Research Inclination is required.

Proper Experiment platform and framework should be discussed.

Lot of sentences need to be revised

Paper needs to be improved. 


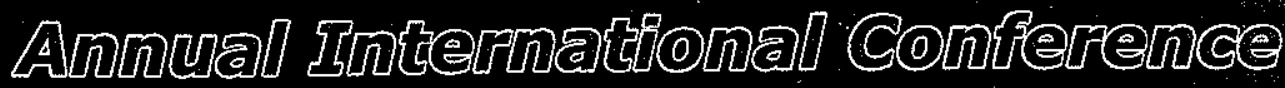 Information Technology Security (IIS) 2010,}

Advanced lopics in Arifilcial lint

and

Network Technologies \& Communications (HiC) 2010

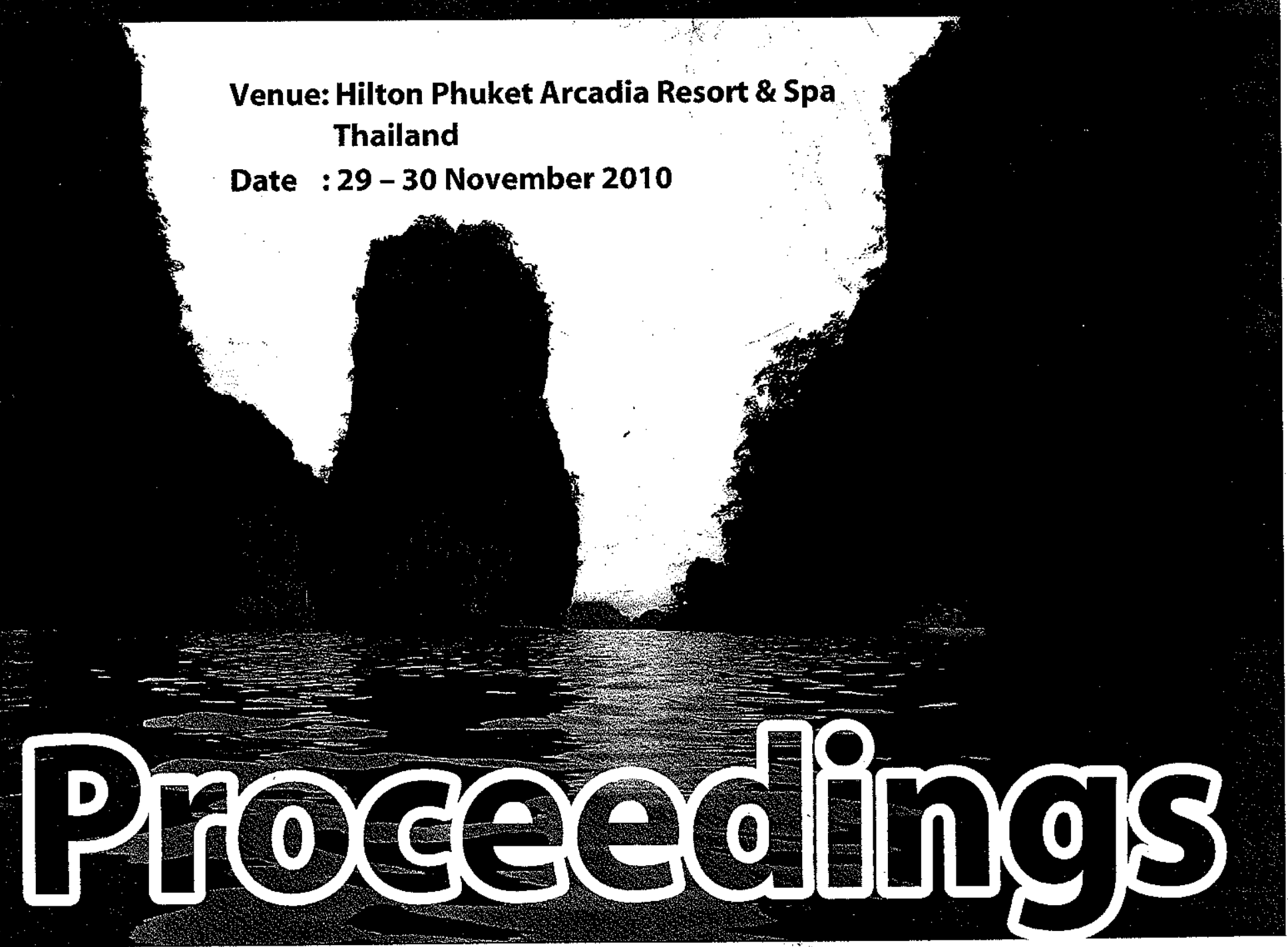

Published and Organized by Global Science and Technology Forum (GSTF)

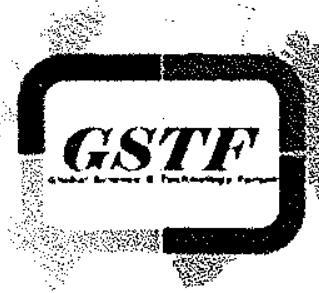

www.globalstf.org 
Proceedings of the Annual International Conference on

$$
\begin{gathered}
\text { Information Technology Security } \\
\text { (ITS 2010); }
\end{gathered}
$$

Proceedings of the Annual International Conference on Advanced Topics in Artificial Intelligence (ATAI 2010)

8

Proceedings of the Annual International Conference on Network Technologies \& Communications (NTC 2010)

Date: 29-30 November 2010

Venue: Hilton Phuket Arcadia Resort \& Spa,

Thailand

\section{PROCE D I N G S}


Published by

Global Science \& Technology Forum (GSTF)

ITS 2010, NTC 2010 \& ATAI 2010

10 Anson Road

International Plaza

Singapore-079903

Tel: $+6563270165 / 66$

Fax: +6563270162

ITS 2010 -E-mail: info@itsecurityconf.org

NTC 2010 - E-mail: info@networkcomm.org

ATAI 2010 - E-mail:info@aiconf.org

Proceedings of the Annual international Conference on

INFORMATION TECHNOLOGY SECURITY (ITS 2010);

Proceedings of the Annual International Conference on

ADVANCED TOPICS IN ARTIFICIAL INTELLIGENCE (ATAI 2010)

\&

Proceedings of the Annual international Conference on NETWORK TECHNOLOGIES \& COMMUNICATIONS (NTC 2010)

Copyright $\odot$ GSTF 2010. All rights reserved.

Published by GSTF and Indexed by EBSCO.

This book, or parts thereof, may not be reproduced in any form or by any means, electronic or mechanical, including photocopying, recording or any information storage and retrieval system now known or to be invented, without written permission from the Publisher.

ISBN: 978-981-08-7654-8 


\section{Pretoce}

We are proud to welcome you to the proceedings of the Annual International Conference on Information Technology Security (ITS) 2010, Annual International Conference on Advanced Topics in Artificial Intelligence (ATAI) 2010 and Annual International Conference on Network Technologies \& Communications (NTC) 2010 held on 29-30 November 2010 at the Hilton Phuket Arcadia Resort \& Spa, Thailand.

The ITS, ATAI and NTC 2010 Conferences continuously aim to foster the growth of the Information Technology industry and its benefits to the community at large. The scientific content of the conference has attracted immense attention and the wealth of information spread across the papers would be extremely useful to the professionals working in the related fields.

It is with great pride and honour that I announce the participation of expert speakers from various countries in this two-day event. This truly is a unique platform for all stakeholders such as researchers, users, technology developers and distributors, and policy makers to discuss, deliberate and exchange experiences.

The Conference Proceedings documents the presentations made at ITS, ATAI \& NTC 2010 and in total this volume contains nearly 50 papers, the end result of a tremendous amount of creative work and a selective review process. We have received research papers from distinguished participating scientists from various countries.

As we have been receiving notable contributions this year, there will be a "2010 BEST PAPER AWARD" and "2010 BEST STUDENT PAPER AWARD" for each of the co-located conferences to recognize outstanding contributions and research publications.

I want to thank all the authors who submitted papers for their participation. They contributed a great deal of effort and creativity to produce this work, and I am happy that they chose ITS, NTC and ATAI 2010 as the place to present it. Credit also goes to the Program Committee members, who donated enormous blocks of time from busy schedules to carefully read and evaluate the submissions.

I would also like to thank the Conference Chair, Professor the Hon. Dr. Stephen Martin the Editors in Chief of ITS, NTC and ATAI, respectively, for contributing towards the success of the conference.

The Organizing Committee would like to take this opportunity to extend our sincere thanks to the Supporting Organizations for their support and encouragement to make the event a success.

Mr. Anton Ravindran

ITS, ATAI \& NTC 2010 Conference Organizing Chair 
This volume of conference proceedings contains a collection of technical research papers presented at the Annual International Conference on Information Technology Security (ITS) 2010, Annual International Conference on Advanced Topics in Artificial Intelligence (ATAl) 2010 and Annual International Conference on Network Technologies \& Communications (NTC) 2010 held on 29-30 November 2010 at the Hilton Phuket Arcadia Resort \& Spa, Thailand.

The ITS, ATAI \& NTC 2010 Conferences are international events for the presentation, inferaction, and dissemination of new results relevant to IT security, network technologies and artificial intelligence, and related areas. As Conference Chair of this event, I would like to express my sincere thanks to all those who submitfed papers for review and those who provided manuscripts for publication in these proceedings.

A special thanks to all our speakers and attendees for making ITS, ATAI and NTC 2010 a successful platform for the industry, fostering growth, learning, nefworking and inspiration.

We sincerely hope you find the conference proceedings to be enriching.

Professor the Hon. Stephen Martin ITS, ATAl \& NTC 2010 Conference Chair 


\section{Annual International Conference on \\ Network Technologies \& Communications \\ (NTC 2010)}


It is our pleasure to present to you the Proceedings of the Annual International Conference on Net-
work Technologies \& Communications (NTC 2010).

The goal of the NTC 2010 is to provide opportunities for academics, researchers, experienced professionals and business people to share their knowledge of network technologies and communications. tion. To meet these requirements, ind wh whomever or whatever they want, regardless of time or locacommunications technologies, each withy has invested heavily in a variety of wireless and wireline seen the emergence of $3 \mathrm{G}$ and $4 \mathrm{G}, \mathrm{WiFi}$ and WiMax band, Powerline and Free space optical.

Next-generation services require unified and scalable technologies spread across the network protocol layers facilitating a converged service model across all partitions of the network. Because of the importance of this technology, decisions of purchase, structure, and operation of networks, manage-
ment has a critical need for understanding the technology of networks and communications.

The novelty of this conference manifests both in its context as well as content. In terms of the content, this conference certainly merits a leading position as most up-to-date research results using cutting-
edge technologies were presented.

I would like to take this opportunity to thank the authors for the care with which they prepared members, organising committee members and especially all the conference participants committee this conference a success. We hope you enjoy reading the proceedings of NTC 2010 .

Dr. A. Kannammal

Associate Professor

Department of Computer Applications, Coimbatore Institute of Technology

India 


\section{General Chair}

Prof. the Hon. Dr. Stephen Martin

Executive Chairman (Pro Tem), Global Science \& Technology Forum

Former Speaker Parliament of Australia

Former Deputy Vice Chancellor (Strategy and Planning) Curtin University of Technology

Former Pro Vice Chancellor International, Victoria University

\section{Program Chair}

Dr. Minhua Ma

School of Computing, University of Derby, United Kingdom

Co-Editors / Reviewers-in-Charge

Dr. Fahim Akhter

Associate Professor, College of Information Technology, Zayed University, Dubai

Dr. A. Kannammal

Associate Professor Department of Computer Applications, Coimbatore Instifute of Technology, India

\section{Program Committee}

\section{Prof. Han-Chieh Chao}

Dean, Electronic Engineering, Institute of

Computer Science $\mathcal{E}$ Information Engineering,

National Ilan University, I-Lan, Taiwan

Prof. Lambert Spaanenburg

Silicon Systems, Lund University, Sweden

\section{Prof. Péter Szolgay}

Professor and vicedean, Faculty of Information Technology, Pázmány Pter Catholic University, Hungary

Assoc Prof. Ibrahim Kamel

Department of Electrical and

Computer Engineering, University of Sharjah, UAE;

Adjunct Professor, CIISE, Concordia University, Canada

Dr. Santi Prasad Maity

Associate Professor, Dept. of Information Technology, Bengal Engineering \& Science University, Shibpur, Howrah-3, West Bengal, India

Dr. Chiranjeev Kumar

Associate Professor, Department of Computer Science $\mathcal{E}$ Engineering, Indian School of Mines (ISM), India
Dr. Maninder Singh

Associate Professor, Computer Science and

Engineering Department, Thapar University, India

Dr. A K Verma

Assistant Professor, Department of Computer Sc. \& Engg., Thapar University, India

Dr. Rajkumar Buyya

Founder and CEO, Manjrasoft Pty Ltd, Melbourne, Australia

Dr. Ziyad.T.Abdul-Mehdi

Faculty of Information Technology,

Multimedia University, Malaysia

Dr. Lei Shu

Department of Multimedia Engineering, Graduate School of Information Science and Technology,

Osaka University, Japan

Dr. Robert C. H. Hsu

Computer Science and Information Engineering, Chung Hua University, Taiwan 
Preface

Foreword

iii

Editorial

iv

Technical Committee

$N$-iii

$N-i v$

\section{Annual International Conference on Network Technologies \&
Communications (NTC 2010)}

\section{Image processing using Al}

A Novel QoS Multicast Routing Protocol in MANETs

Mohammad M. Qabajeh, Aisha H. Abdalla, Othman Khalifa and Liana K. Qabajeh

A Scalable and Secure Position-Based Routing Protocol for MANETs

Liana Khamis Qabajeh, Miss Laiha Mat Kiah and Mohammad Moustafa Qabajeh

A New Optical Switching Network Architecture with Crosstalk Free And Nonblocking

Manjur Ahmed, Mostafijur Rahman, Ahmad Nasir Che Rosli, M. M. Hafizur Rahman, Fareq Malek, Md.
Sakoat Hossen and R. B. Ahmad

Modeling Road Traffic Networks for Control

T. Peter and J. Bokor

Study and Implementation for the Architecturelayer of LonTalk Protocol
Su Goog Shon, Soo Mi Yang and Jae Jo Lee

Microwave Generation Based on an Inverse-Gaussian Apodized Fiber Bragg Grating and a Semiconductor Optical Amplifier Operating in Low-Gain Regime

Bo Lin, Swee Chuan Tjin, Han Zhang, Dingyuan Tang, Jianzhong Hao, Bo Dong and Sheng Liang

Towards Stable Routes in Dense Manets

Sharmila Sankar and V. Sanakaranarayanan

A Base Station Co-operation Protocol for Indoor Base Stations

Akindele Segun Afolabi, Chikara Ohta and Hisashi Tamaki

Hybrid Predictive Modeling for Multivariate Responses

S. Tom Au, Rong Duan, Guangqin Ma and Rensheng Wang

Weless Device Location Sensing In a Museum Project

Wony Anwar and Priyadarsi Nanda

Efficiency Development of Performance Management System for Base Stations

Narangsan Suracharoenchaikul and Namkhun Srisanit

N-55 
A Hash-free Overlay with Eventually Optimal Locality Martin Raack, Christian Wuertz, Philipp Berndt and Odej Kao

Performance Analysis of LEDBAT in the Presence of TCP-Newreno

Distributed Cache Framework and Its Data Procurement Algorithm on Global Data Grids

Spanning a Global Delay Model from HTTP-Measurements

Philipp Berndt, Martin Raack and Odej Kao

Study of 0.2-nm Channel Spacing WDM System

N. Ahmed, S. A. Aljunid, R. B. Ahmad, Hilal Adnan Fadil and M. A. Rashid 